\title{
Gulliver's Travels (Libro III) \\ La sátira y su traducción
}

MAgdalena LóPez PÉRez

Universidad de Córdoba

Resumen: Jonathan Swift se caracteriza por sus abundantes escritos satíricos, entre los que se encuentra su obra más importante y reconocida, Gulliver's Trave/s. Su estilo y lenguaje propios confieren a dicha obra una sólida unidad, mediante la cual consigue inducir al lector a la contradicción y convencerle de la historia, aún siendo evidente la imposibilidad natural del hecho que narra. Sin embargo, tales contradicciones satíricas no siempre son recogidas en las diversas traducciones que se han realizado de esta obra.

Abstract: Jonathan Swift is characterized by his numerous satirical writings, among which we can find his most important and recognized work, Gulliver's Travels. His own style and language provide the book with a solid unity, by means of which Swift persuades the reader and makes him believe that the story is completely true, even though it is evident the natural impossibility of the told events. However, such satirical contradictions are not always present in the several translations that have been made of this work

Palabras clave: Sátira. Traducción. Prosa literaria.

Key words: Satire. Translation. Literary prose.

1. VIDA

Jonathan Swift (1667-1745), novelista, poeta y escritor satírico irlandés, nace en Dublín el 30 de noviembre. Segundo y único hijo varón de Jonathan Swift y Abigaile Erick Swift (ya contaban con una hija, Jane), Swift nació siete meses después de que su padre muriera. Recibe educación en Kilkenny School y en el Trinity College de Dublín gracias a la ayuda de sus tíos. Tras seguir sus estudios en la Universidad de Dublín (1681-1688), abandona Irlanda para reunirse con su madre que residia en el condado de Leicester. En 1689, comienza a trabajar como secretario de William Temple, importante hombre de estado de la época, miembro del parlamento y destacado diplomático, que será su protector durante diez años. Sería durante este periodo cuando desarrollaría su aprendizaje político durante los años 1689-1694 y 1696-1699. 
Sus nuevas funciones como secretario le permiten realizar los estudios de teología que termina con el doctorado en 1692. En 1674 Jonathan Swift es nombrado pastor en Kilroot, cerca de Belfast. Alli sólo se quedará unos meses para marcharse después a Moon Park donde vive Sir William Temple. Para defender a Temple en su famosa controversia con el eminente clasicista Richard Bentley, Swift pone en circulación una breve parodia épica, The Battle of the Books (1697), sobre el enfrentamiento entre los defensores de la Antigüedad, a los que favorece, y los defensores de la Modernidad. En Moon Park trabaja como preceptor de Esther Johnson, legalmente hija de un intendente, pero, muy probablemente, hija ilegítima de Temple y de su ama de llaves. Swift se encuentra con ella por primera vez cuando ésta tenía ocho años y comienza a enseñarle a leer. Esther, a la que el autor llama cariñosamente Stella, despierta en Swift una larga y profunda pasión. Sin embargo, poco se sabe de esta relación, tan enigmática como extraterrenal, pero muy apasionada, según se deduce de sus escritos en Journal to Stella. Algo parecido sucedió con Esther Vanhomrigh, a quien el autor llamaba Vanesa, como queda reflejado en su Cadenus and Vanessa.

En 1694, regresa a Irlanda, donde es ordenado sacerdote. Tras reconciliarse con Temple, del que se separó por ciertas diferencias, vuelve a su servicio en 1696. Supervisa la educación de la hija de la recién enviudada hermana de Temple, y permanece con el caballero hasta su muerte, en 1699. Durante ese tiempo, tuvo frecuentes discusiones con su patrón, aunque dispuso de mucho tiempo para la lectura y la escritura. Tras la muerte de Sir William Temple, Swift regresa de nuevo a Irlanda donde se instala en 1700.

Pronto obtiene el beneficio eclesiástico de Lavacor en el condado de Meath y una prebenda en la Catedral de San Patricio en Dublín. En 1701 publica su primer panfleto político, donde toma claramente partido por los 'Whigs', partido político de corte progresista que representaba a la población urbana (industriales, comerciantes y banqueros) y que consideraban esencial la autoridad del Parlamento, no apoyaban el monopolio de la iglesia anglicana y deseaban participar en los asuntos de Europa. Se siente cada vez más atraído por el mundo de la política, hasta tal punto que su carrera política abarca un período de cuarenta años y tres monarcas, la reina Anne y los reyes George I y George II.

En 1710 sube al poder en Inglaterra el partido 'Tory' y Swift se pasa rápidamente a sus filas, defendiendo ahora a los conservadores, terratenientes de origen nobiliario y clérigos que creian en el poder supremo de la Corona, cuya pretensión máxima era que se persiguiera a los protestantes no anglicanos y que no se inmiscuyeran en los asuntos extranjeros. Comienza a dirigir entonces sus ataques contra los 'Whigs', a 
través de una serie de textos cortos. Fue en ese mismo año de 1710 cuando inicia Journal to Stella, escrito en 1710 y 1711.

Entre 1711 y 1714 dirige el Examiner, órgano informativo de los 'tories', y publica muchos panfletos, en los que defiende abiertamente la política social del gobierno 'Tory', predisponiendo a la opinión pública a la paz con Francia. De entre esos textos, el más elocuente e influyente fue On the Conduct of the Allies, publicado en noviembre de 1711, un inteligente panfleto que preparó el camino para que el gobierno 'tory' se retirara de la alianza que tenía con otros países europeos en contra de Francia en las guerras de Sucesión española. Dicho panfleto fue también la causa de la dimisión de John Churchill, primer duque de Malborough, comandante en jefe de las Fuerzas Armadas británicas.

En 1713 es nombrado Deán de la Catedral de San Patricio, aunque no puede acceder al obispado puesto que su The Tale of a Tub (1704), una agudisima sátira contra los abusos de la religión y el saber, no habia gustado a la reina Ana al atacar tanto a los anglicanos como a los disidentes y a los católicos romanos. La caída de los 'Tories' en 1714 hizo definitivo su exilio voluntario en Irlanda. Desde entonces se dedica a defender con ardor a su país y publica un ingente número de obras políticas hasta su muerte en 1845. En ese mismo año (1713), Jonathan Swift se convierte en miembro fundador del 'Club Scriblerus' junto a Pope, Arbuthnot, Gay, Parnell, Congreve, Lord Oxford y Attenbury, entre otros.

En 1717, ocupa por fin su cargo de Deán de la Catedral de San Patricio de Dublín. Entre 1724 y 1725 publica anónimamente Drapier's Letters, una serie de panfletos en los que defendía la validez de la moneda irlandesa, y que ocasionaron el fin de la fraudulenta patente otorgada por la corona a un comerciante inglés para acuñar monedas en Irlanda. Estos panfletos le hicieron muy popular entre sus conciudadanos. Con el mismo propósito pro-irlandés escribe $A$ Modest Proposal: For Preventing the Children of Poor People in Ireland from Being a Burden to Their Parents or Country, and for Making Them Beneficial to the Public (1729), su sátira más poderosa y penetrante. Esta propuesta especialmente irónica defiende la postura de que los niños irlandeses pobres podian ser vendidos como carne para mejorar la dieta de los ricos. Su obra maestra, Gulliver's Travels, fue publicada como obra anónima en 1726 aunque nadie ignoraba que Swift era su autor.

El estilo y el lenguaje de Swift proporcionan una sólida unidad de tono y de significación a la obra. Decantándose por la ironía, Swift consigue inducir al lector a la contradicción y convencerle de la historia, aún siendo evidente la imposibilidad natural del hecho que narra. Durante los seis años que tardó en escribirla, el autor va añadiendo ciertas reflexiones acerca de la naturaleza humana. Con una narración imaginativa, ingeniosa y sencilla 
de leer, esta devastadora sátira sobre la corrupta clase dirigente inglesa alcanzó un profundo éxito, hasta el punto de convertirse en un referente de texto irónico, ya que contiene todas las caracteristicas que W.A. Eddy propone, como podemos ver en el siguiente párrafo:

"Irony, it is held, is a negative attitude, irreverent to ideals, discouraging to good works, harsh and unsympathetic to the amenities of life. Satirists are a bilious breed who void their rheum boorishly over expensive rugs and evening dresses [...] Crude and violent, satire does not dwell with beauty; it mars rhyme and sours fiction in the same way that caricatures distorts the features of a portrait. At best it is candid and corrective; but the moral it teaches is cynical and pessimistic, the laugh it raises is bitter and unkind"1.

Sus últimos años, tras la muerte de Stella, se caracterizan por una serie de indicios de demencia. Sufre ataques de vértigo $y$, tras un largo periodo de deterioro mental, fallece el 19 de octubre de 1745, dejando todas sus posesiones a organizaciones benéficas. Enterrado en la catedral de la que fue deán, junto al sepulcro de Stella, su epitafio, escrito por él mismo en latín, reza asi:

"Hic depositum est corpus JONATHAN SWIFT S.T.D. Huyus Ecclesiae Cathedralis Decani ubi saeva indignatio ulterius cor lacerare nequit. Abi Viator et imitare, si poteris Strenuum pro virili Libertatis Vindicatorem"

Obiit 19 Die Mensis Octobris A.D. 1745 Anno

William Butler Yeats realizó la traducción al inglés de dicho epitafio como sigue,

"Swift has sailed into his rest. Savage indignation there cannot lacerate his breast. Imitate him if you can, world-besotted traveler. He served human liberty".

\section{LA SÁTIRA EN LAS OBRAS DE SWIFT}

La característica más destacada de su vida entera y de su obra, es un compromiso constante con la honestidad y la verdad tal como él la entendió. Swift dedicó toda su energía y su extraordinaria habilidad verbal y literaria "a indagar y proponer verdades de orden moral, a transformar en símbolos y

\footnotetext{
${ }^{1}$ Eddy, WILliam ALfRed (ed.), Swift: Satires and Personal Writings. London: OUP, (1932), 1973, pp. xxii-xxiii.
} 
metáforas las obsesiones y desilusiones que le inspiró la naturaleza humana" .

De hecho, Swift mantenia siempre una preocupación constante por el lenguaje, objeto, por otra parte, de una observación mordaz y de intencionadas sátiras en los dos últimos viajes. Normalmente, Swift utiliza un estilo arrogante en sus escritos y dicta más que persuade. En lugar de ser acusado de utilizar un tono demasiado jocoso en sus escritos, debido, principalmente, a este lenguaje altivo y jactancioso, fue, por el contrario, halagado por esta arrogancia y soberbia.

La obra de Swift, aunque escasa en narrativa, es muy numerosa y abundante en poemas, correspondencia, panfletos y tratados políticos, religiosos y sociales. Sin embargo, su obra siempre ha estado rodeada por la polémica, entre otras el significado último de sus escritos o ciertos hechos de su vida y sus relaciones personales y profesionales.

Esta polémica está presente fundamentalmente en sus escritos de carácter satirico, tal y como A.C. Elias Jr. manifiesta en el siguiente párrafo:

"...Swift is probably the hardest for modern critics to cope with, not only because he habitually mocks all that we academics hold dear but also because he affects us in ways which our training leaves us handicapped in dealing with. When we teach ourselves to read and analyze literature with professional dispassion, we find ourselves hamstrung when dealing with an author skilled at playing tricks with his readers- provoking their laughter in unsettling ways, stirring them up obscurely, making fools of them. Before we can analyze the literary ways and means of Swift's satire, we may find that we need to confess how badly we've been stung by it, an awkward position at best, and for intellectuals a well-nigh intolerable one ... It is little wonder that Swift criticism has demonstrated such bewildering variety and vehemence over the years..."3.

Durante toda su vida e incluso tras su nombramiento como deán de la Catedral de S. Patricio, Swift continuó escribiendo de manera satírica tanto en prosa como en poesía, utilizando diferentes registros para atacar aspectos personales, de conducta, filosóficos, políticos, religiosos, cívicos y otros:

"The opinions held by Swift, though expressed with uncommon vigour, reflect from the very age and body of the time his form and pressure" ${ }^{4}$.

\footnotetext{
${ }^{2}$ SWIFT, JONATHAN, Los viajes de Gulliver (introducción de Aránzazu Usandizaga, traducción de Begoña Gárate Ayastuy). Madrid: Alianza, 1994, p. 8.

${ }^{3}$ ELIAS, A. C., Swift at Moor Park: problems in biography and criticism. Philadelphia: University of Pennsylvania Press, 1982, p. $x$.

${ }^{4}$ EDDY, W. A. (ed.), Swift: Satires and Personal Writings, p. xiv.
} 
Entre los años 1696-1699, Swift escribe dos de sus obras más importantes: A Tale of a Tub, en la que defiende la posición intermedia de lás iglesias anglicanas y luteranas e incluye multitud de referencias a autores antiguos y también algunas de ellas a autores modernos, y Battle of the Books, tomando partido por los Antiguos (aquellos que creían en la superioridad de los clásicos y las humanidades) contra los Modernos (aquellos que apoyaban la superioridad de la ciencia moderna, del aprendizaje moderno, de la política moderna y de la literatura moderna).

The Journal to Stella (1710-1713), una serie de cartas escritas por Swift a Esther Johnson, incluye el poema "The Windsor Prophecy", un ataque satírico acerca de la persona y personalidad de la Duquesa de Somerset, una ayudante pelirroja de la reina Ana que no sentía mucho cariño por Swift debido a los despectivos comentarios que el autor había escrito sobre su familia.

En A Modest Proposal (1729), una reacción contra las prácticas comerciales inglesas que impactaron negativamente en Irlanda, Swift escribió uno de los mejores trabajos irónicos en lengua inglesa. En este ensayo de carácter eminentemente satírico, Swift propone una posible manera de solventar el problema de la miseria entre los católicos de Irlanda. Su propuesta consistia en hacer engordar a los niños desnutridos para que sirvieran de alimento a los ricos terratenientes irlandeses. Los hijos de los más pobres podrían ser vendidos en el mercado de la carne cuando tuvieran tan sólo un año y, de esta manera, se combatiría la superpoblación y el desempleo, las familias reducirían el gasto tan costoso de criar a sus hijos, al mismo tiempo, que recibirían una paga extra, se mejoraría la experiencia culinaria de los ricos y se contribuiría al bienestar económico de la nación.

Del mismo modo, ofrece no sólo un estudio estadístico de sus afirmaciones, sino también datos especificos sobre el número de niños que se deberían vender, su peso y precio. Sugiere también algunas recetas para preparar esta delíciosa y novedosa carne y nos anticipa que la práctica de vender y comer niños tendrá efectos positivos en la moralidad familiar: los esposos tratarán a sus mujeres con mayor respeto y los padres valorarán a los niños de una manera hasta ahora desconocida. Su conclusión es que la puesta en marcha de este proyecto se centrará más en solucionar los complejos problemas sociales, políticos y económicos de Irlanda que en ninguna otra medida que se hubiera propuesto.

The Drapier's Letters (1724) es la respuesta de Swift a la continua subyugación llevada a cabo por Inglaterra de todos aquellos que viven en Irlanda. Dichas cartas suscitaron tanta oposición que los ingleses ofrecieron una recompensa de $£ 300$ por el nombre del autor. Sin embargo, los 
irlandeses nunca le traicionaron, pese a saber que el autor de las cartas había sido Jonathan Swift. En su lugar, lo convirtieron en héroe nacional.

Su novela más reconocida, Gulliver's Travels (1726), es una continua sátira del ser humano y su sociedad, como las debilidades, los vicios y las locuras inherentes a los seres humanos. Esta sátira alcanza su momento máximo en la comparación de Swift entre los 'Houyhnhnms' (caballos) y los 'Yahoos' (criaturas parecidas a los humanos) en el Libro IV. Esta obra se publicó bajo un profundo silencio ya que ni tan siquiera el editor conocía el autor de la obra, puesto que se le envió a su casa en un coche de alquiler. Gulliver's Travels fue un éxito inmediato: Voltaire (1694-1778), que estaba en ese momento viviendo en Inglaterra, lo tradujo al francés, haciendo que se conociera más allá del canal.

\section{GULLIVER'S TRAVELS: THE THIRD BOOK}

Gulliver's Travels fue una obra maestra escrita, en mayor o menor medida, para satisfacer un encargo. El Club 'Martinus Scriblerus', compuesto por destacados personajes como Pope, Arbuthnot, y Gay, propuso satirizar las locuras, vicios, pedantería y el falso saber de los hombres modernos, eruditos y científicos. La correspondencia establecida entre Swift y sus amigos prueba que

"in his social life he was not as abnormal as sometimes has been alleged. Like many strong-minded men, his intimate friends were few; but these few were his for life. Many of his letters are excellent models of the easy familiar style. Some throw light on his literary practice and his moral philosophy. Others are full of glimpses into the petty economy of his domestic life, especially after his exile to Ireland: the weekly round of restful pleasures and inconsequential irritations. But though written to intimate friends in a personal, non-ironic style, we do not find the whimper and sob of a lonely heart any more than we find them in the Journal, or in Gulliver. Men at that time did not dip their pens in tears least of all did Jonathan Swift. His reserve was not so much pathological as it was logical, normal, and expected"

A cada uno de los miembros del grupo se le dio un tema, y el de Swift consistió en satirizar los numerosos y populares volúmenes que describían los viajes hacia tierras lejanas. Pasaron diez años entre el proyecto 'Scriblerus' y la publicación de los Trave/s, pero cuando Swift acabó la obra, habia realizado no sólo lo que se han convertido en un clásico para los niños (en su edición resumida) sino también una obra ejemplar satírica.

\footnotetext{
${ }^{5}$ EdDY, W. A. (ed.), Swift: Satires and Personal Writings, p. xx.
} 
Cuando Swift publica Gulliver's Travels es un experto en el arte de la parodia y de la alegoría, y escoge algunos de sus juegos estilisticos favoritos a la hora de describir las fantásticas aventuras que le suceden al protagonista. Por otra parte, tal y como Usandizaga señala, "estos viajes son también una obra que se inscribe y se inspira en una tradición bien conocida en la historia de la narrativa inglesa. El género más popular después de la literatura religiosa en el s. XVII, es la literatura de viajes"6.

Swift mantuvo la forma de los libros de viajes pero amplió su objetivo. En lugar de parodiar simplemente la literatura de viaje, decidió atacar lo que consideraba que eran los vicios más destacados de las personas. Convirtió lo abstracto en concreto, al mismo tiempo que las ideas iban tomando forma en grotescas y extranjeras criaturas, las costumbres absurdas se representaban mediante objetos absurdos, convirtiendo así a lo familiar en algo verdaderamente nuevo y sorprendente.

Gulliver's Travels es una historia de aventuras, en realidad una historia de aventuras desgraciadas que incluye los viajes de Lemuel Gulliver, oficial médico de una embarcación, que, debido a una serie de contratiempos en el itinerario previsto, acaba desembarcando en unas islas desconocidas y viviendo con una serie de personas y animales de tamaño muy inferior al suyo y con unos comportamientos y maneras de pensar un tanto inusuales. Finalmente, consigue regresar a su hogar en Inglaterra en donde se recupera de estas experiencias tan poco corrientes para volver a embarcarse en otro nuevo viaje.

Sin embargo, Swift no fue el primer autor que envía a su héroe de viaje a lugares imaginarios, aconteciendo innumerables historias fantásticas. La ficticia obra de Daniel Defoe, Robinson Crusoe, publicada en 1719, se basaba ligeramente en la verdadera historia de Alexander Selkirk. Otros precedentes de este tipo de viajes fantasiosos fueron las obras de Bergerec, Histoire Comique des États et Empires de la Lune et du Soleil (1657) y la Utopia de Thomas More (1516).

Son muchos los autores que han considerado la posible existencia de alegorías en Gulliver's Travels de Swift. Por ejemplo, Emilio Lorenzo habla de claras coincidencias entre el último periodo de reinado de la reina Ana y la primera parte de dicha obra. Del mismo modo, mantiene que
"aunque al Emperador de Liliput se le encuentra parecido, discutible, con Jorge I. El reino de Blefuscu es, sin duda, Francia, y en los personajes de la corte de Liliput se han visto paralelos con destacadas figuras de la corte de la reina Ana y de Jorge I. Asi, Flimnap, principal enemigo de Gulliver en la corte, seria Sir Robert Walpole, jefe de

\footnotetext{
${ }^{6}$ SWIFT, JONATHAN, Los viajes de Gulliver (introducción de Aránzazu Usandizaga, traducción de Begoña Gárate Ayastuy), p. 11.
} 
gobierno con el partido whig casa ininterrumpidamente desde que cayó Robert Harley (el gran protector tory de Swift) hasta el mismo año en que Swift pierde sus facultades mentales; para Redresal, que figura como aliado influyente de Gulliver, se ha encontrado en la vida de Swift a Lord Carteret, amigo suyo, y a Lord Lieutenant de Irlanda, y a Lord Townshend, secretario de Estado y adicto a Walpole; Bolgolam seria el conde de Nottingham, que, aunque tory, se enfrentó con Harley y su gobierno; la disputa sobre el modo de cascar los huevos satiriza la polémica entre católicos y protestantes; aludidos quedan, aparentemente, Enrique VIII, que hizo a sus súbditos seguirle en su conflicto con Roma, Carlos I, que fue ejecutado, y Jacobo II, que fue destronado; de igual manera se ve en la caída en desgracia de Gulliver con su Soberana una réplica literaria del tropiezo que sufrió Swift en su carrera eclesiástica por la grosería y desenvoltura que mostró en A Tale of a Tub, y que le costaron el favor de la reina Ana".

Pollux Hernúñez, por su parte, señala la existencia de tres niveles en la obra, como podemos deducir del siguiente fragmento:

"Gulliver's Travels funciona en tres niveles: la pura anécdota, la sátira histórica y la sátira universal. La anécdota es simplemente la serie de aventuras que Gulliver corre en los diversos paises que visita [...] La sátira histórica se ve tanto en el género y estilo (Swift se burla de las convenciones de la novela de aventuras) como en el contenido: la mayor parte del primer viaje es un roman-à-clé en el que Swift fustiga, bajo el disfraz de la ficción, a personajes reales [...] La sátira universal es la que se refiere al hombre en general" ${ }^{8}$

Para nuestro propósito, nos centramos en el apartado satírico de Gulliver's Travels y, más concretamente, en el libro tercero de sus aventuras, cuando el protagonista se marcha de viaje a Laputa, a Balnibarbi, Glubbdubdrib, Luggnagg y a Japón, para realizar, posteriormente, un estudio y análisis de distintos fragmentos desde un punto de vista traductológico.

Tras sus aventuras en Lilliput y en Brobdingnag, Gulliver emprende, en la tercera parte del libro, un nuevo viaje con rumbo a oriente. Tras llegar a una isla, Gulliver es nombrado capitán de una expedición para visitar las islas más cercanas y establecer el comercio. Es en esta aventura cuando varios piratas atacan la expedición y dejan a Gulliver en un pequeño bote en el mar para que se defienda solo. Es de esta manera como, mientras

\footnotetext{
${ }^{7}$ SWIFT, JONATHAN, Los viajes de Gulliver (traducción, introducción y notas de Emilio Lorenzo). Cáceres: Clásicos de la Lengua Inglesa en Traducción, 1998, p. 16.

${ }^{8}$ SWIFT, JONATHAN, Los viajes de Gulliver (traducción, notas y apéndice de Pollux Hernúñez). Madrid: Anaya, 1999, p. 348.
} 
navega a la deriva por el mar descubre una isla voladora llamada Laputa. Una vez allí, Gulliver conoce a sus habitantes, incluyendo al rey, todos ellos muy preocupados con todo aquello que esté directa o indirectamente relacionado con las matemáticas y la música. Además, los astrónomos se sirven de las leyes magnéticas para desplazar la isla de arriba abajo, de izquierda a derecha, y hacia otros lados, con el fin de controlar los movimientos de la isla en relación con otra isla que se encontraba un poco más al sur, Balnibarbi. Gulliver visita, asimismo, esta última isla además de las islas de Glubbdubdrib y la de Luggnagg. Finalmente, Gulliver llega a Japón donde se reúne con el emperador japonés. Desde allí se dirige a Ámsterdam y, por último, regresa de vuelta a Inglaterra.

El ensimismamiento de Swift es la causa de su inoperancia y su sátira se dirige ahora más directamente contra el racionalismo, la 'Royal Society' y, en general, contra la pasión pedante, exagerada y en muchos casos inútil por la ciencia. En Laputa, los habitantes investigan cómo obtener rayos de sol a partir de los pepinos, e intentan transformar los excrementos humanos en los alimentos de los proceden o convertir el hielo en pólvora, o labrar la tierra con cerdos.

Ahora bien, como señala Usandizaga, "a pesar de que la intención satírica de Swift en esta primera parte del tercer viaje es evidentemente la de burlarse una vez más de la vanidad humana, en este caso de su vanidad intelectual, que hace de los habitantes de Laputa y de Balnibarbi unos incapaces en todos los aspectos de la vida práctica, las referencias políticas son también en este viaje considerables, y las relaciones entre la isla y el continente se suelen entender como metáfora de las problemáticas y complejas relaciones entre el rey y el Parlamento en la Inglaterra de principios del s. XVIII"'.

De las cuatro partes de Gulliver's Trave/s, la tercera, "A Voyage to Laputa", ha sido, para muchos, la parte menos satisfactoria y entendida. Son muchos los críticos que entienden la tercera parte como una serie completa de episodios que carecen de sentido y artificios propios, en comparación con el resto de la obra, aunque quizás Swift haya pretendido que esta parte parezca fragmentada, poco natural e incluso incoherente. No hayamos ahora no ante una combinación exhaustiva de nuevas ciencias y filosofías, sino más bien ante un consejo y advertencia para mantener la vista en el pasado ante esta nueva era extremada e imprudentemente optimista.

Las mayores críticas a las que esta parte ha tenido que hacer frente han sido aquellas relacionadas con las curiosas combinaciones de las

\footnotetext{
${ }^{9}$ SWIFT, JonATHAN, Los viajes de Gulliver (introducción de Aránzazu Usandizaga, traducción de Begoña Gárate Ayastuy), p. 15.
} 
matemáticas y la música de los laputienses, con la falta de unidad de los Balnibarianos y, especialmente, con los experimentos en la Gran Academia de Lagado y con el mágico invento de la Isla Voladora. Sin embargo, también debemos tener en cuenta que, pese a que tras una primera lectura pueda parecernos un trabajo exclusivamente rocambolesco, Swift extrae todo su material satírico de novedosos artículos. La mayoría de las ideas que presenta están basadas en experimentos reales narrados en la literatura de sus días $y$, particularmente, en informes publicados en las "Transacciones Filosóficas de la Sociedad Real" durante el último tercio del s. XVII y el primer cuarto del s. XVIII incluyendo, asimismo, el material publicado en 1726, el año en el que Swift compuso la tercera parte.

Cierto es también que Swift utiliza dicho material extrapolado a términos extremos en conjugación con la actitud prevaleciente por aquel entonces de que todas las matemáticas y el aprendizaje teórico eran inútiles, para difundir su mensaje subyacente de que la virtuosidad de sus dias habia perdido la antigua visión del mundo. Pese a todo, Swift continúa satirizando las nuevas tendencias en matemáticas, en ciencias teóricas y en política, pero, según los críticos, lleva a cabo esta sátira de manera menos decidida a como lo hacía en los otros tres viajes de Gulliver, todo ello debido fundamentalmente al carácter episódico de este tercer viaje, a la multiplicidad de temas y a la falta de una intuición filosófica clara en los viajes hacia Lilliput y Brobdingnag o en los salvajes ataques acerca de la condición humana en el viaje a Houyhnhnyms.

\section{TRADUCCIÓN}

Como hemos mencionado anteriormente, aunque la sátira que aparece en la tercera parte de la obra se sitúe en tres niveles, W.A. Eddy insiste en afirmar que podemos extraer otros dos rasgos bastantes significativos de la sátira que Swift confiere a esta tercera parte al señalar:

"Two features of Swift's satire may be noted here, in passing. The use of fiction as sugar-coating for a pill of bitter philosophy is one of his greatest distinctions [...] Secondly, to this use of fiction must be added Swift's wit and humour -also an ingredient indispensable to good satire everywhere, since the purpose of satire is to ridicule what the writer would demolish"10

Vamos a estudiar a continuación varios pasajes, todos ellos de carácter satírico, centrándonos de una manera más exhaustiva en sus traducciones, con el fin de observar si dicho tono jocoso se mantiene en las

\footnotetext{
${ }^{10}$ EdDY, W. A. (ed.), Swift: Satires and Personal Writings, pp. xxix-xxx.
} 
versiones en español. El primer rasgo característico aparece en la traducción del título de esta tercera parte en la versión de Emilio Lorenzo quien, en vez de traducir el lugar de destino por Laputa (tal y como hacen las otras tres versiones), utiliza el término Lapuda, alegando que en inglés el autor no quería suscitar ningún sentido peyorativo que si aparece en español. Sin embargo, al optar por dicha traducción, Lorenzo no hace sino adaptar la versión inglesa, aunque para ello pierda gran parte del sentido original $l^{11}$. Es el propio autor en el capítulo segundo de esta tercera parte, quien realiza una más de sus parodias lingüísticas, esta vez acerca de los dislates de los etimólogos:

"The Word, which I interpret the Flying or Floating Island, is in the Original Laputa; whereof I could never learn the true Etymology. Lap in the old obsolete Language signifieth High, and Untuh a Governor, from which they say by Corruption was derived Laputa, from Lapuntuh. But I do not approve of this Derivation, which seems to be a little strained. I ventured to offer to the Learned among them a Conjecture of my own, that Laputa was quasi Lap outed; Lap signifying properly the dancing of the Sun Beams in the Sea; and outed a Wing, which however I shall not obtrude, but submit to the judicious Reader" ( $p .156$ )

El siguiente párrafo es una crítica indirecta a la pasión desenfrenada e inculta que los contemporáneos de Swift sentían por la ópera. Para Pitágoras, los cuerpos celestes emitían sonidos armoniosos e imperceptibles al oído humano que simbolizaban el orden divino que el ser humano captaba de una manera tan perfecta. Swift, por su parte, subraya la arrogancia de los laputienses al intentar mejorar esta armonía con acciones vanas e inútiles:

"He said, that the People of their Island had their Ears adapted to hear the Musick of the Spheres, which always played at certain Periods; and the Court was now prepared to bear their Part in whatever Instrument they most excelled" (p. 157)

En cuanto a las traducciones, la versión de Emilio Lorenzo (primera) refleja de una manera más fiel el texto original que las otras versiones, aunque al final del fragmento añade cierta información adicional que no aparece en el texto original. Por su parte, la versión de Pollux Hernúñez (segunda) también realiza una traducción correcta con respecto al original. En cuanto al resto de versiones, la de Gárate, versión tercera, al igual que la

\footnotetext{
11 SWIFT, JONATHAN, Los viajes de Gulliver (traducción, introducción y notas de Emilio Lorenzo). Cáceres: Clásicos de la Lengua Inglesa en Traducción, 1998, pp. 183-184.
} 
versión de Guardia Massó (cuarta) realiza ciertos cambios de tiempo verbal, siendo ésta última la que más dista del texto original de Swif:

"Diciendo que las gentes de la isla tenian adaptado el oido para oír la música de las Esferas, que siempre sonaba en ciertos períodos, y que en aquel momento la Corte estaba preparada para participar concertadamente tocando cada uno el instrumento en que más se distinguía" (p. 184)

“Dijo que la gente de la isla tenía los oidos adecuados para escuchar la música de las esferas, que siempre se oía en ciertas épocas, y que a la sazón los miembros de la Corte estaban dispuestos para participar con cada uno de los instrumentos en los que más sobresalian" (p. 176)

“Dijome que la gente de la isla tiene sus oídos adaptados para escuchar la música de las esferas, que siempre sonaba en determinadas fechas, y que la corte estaba por entonces dispuesta para participar cada cual con el instrumento en que sobresalia" (p. 186)

"La gente de la ișla tiene los oídos adaptados a la música de las Esferas, que siempre se hacia sentir en un periodo determinado y cada uno de los cortesanos sabia acompañar la música celeste con su instrumento favorito" (p. 148)

La sátira científica de los viajes apunta, tal y como Pedro Guardia Massó anota en su introducción de la obra, "a teorias absurdas y estériles como el extraer rayos de sol de los pepinos, el reconvertir los excrementos a su estado primerizo, el fabricar tejidos con hilos de araña o almohadas con mármol triturado. Los laputienses con todos sus conocimientos músicos y matemáticos-astronómicos, llegan al extremo de partir los alimentos en formas geométricas (pan en conos, pechugas de ternera en forma de arpa, etc.). Su ciencia teórica los capacita para tomar las medidas corporales de Gulliver -basándose en complejas operaciones matemáticas - para confeccionarle un traje. Sin embargo, el producto final, el traje confeccionado, es un verdadero desastre. Los laputienses consideran que rebasar los limites de los frios y racionales postulados especulativos implican una degradación intelectual; y esto es hasta tal punto cierto que su idioma carece de términos para expresar los conceptos de inventiva, fantasía e imaginación. Se establece, pues, la antinomia ciencia especulativa, ciencia utilitaria. Para Swift -y para Gulliver- el cienticismo improductivo es pseudociencia"12.

\footnotetext{
${ }^{12}$ SWIFT, JONATHAN, Los viajes de Gulliver (introducción, traducción y notas de Pedro Guardia Massó). Barcelona: Planeta, 1984, p. XVI.
} 
Swift trasluce en sus viajes una obsesión biológica: el término excremento -asi como otros relacionados con las necesidades corporalesaparece una veintena de veces en el texto En la Academia Real de Lagado se intenta transformar los excrementos humanos en el alimento originario y los conspiradores antigubernamentales se descubren mediante el análisis de sus deposiciones.

Estos escritos sobre la Academia representan un ataque frontal a la 'Royal Society' y a su espíritu experimental e innovador. Swift ataca al progreso que va en contra de los dictámenes del sentido común y la razón, pero va más allá en su crítica puesto que estos miembros no son sólo inventores desinteresados, sino que son hombres de Estado con total autoridad para aplicar los proyectos que diseñan. Sin embargo, estos proyectos tienen un único inconveniente:

"The only Inconvenience is, that none of these Projects are yet brought to Perfection; and in the mean time, the whole Country lies miserably waste, the Houses in Ruins, and the People without Food or Cloaths. By all which, instead of being discouraged, they are Fifty Times more violently bent upon prosecuting their Schemes, driven equally on by Hope and Despair: That, as for himself, being not of an enterprizing Spirit, he was content to go on in the old Forms; to live in the Houses his Ancestors had built, and act as they did in every Part of Life without Innovation. That, some few other Persons of Quality and Gentry had done the same; but were looked on with an eye of Contempt and ill Will, as enemies to Art, Ignorant, and ill Commonwealthmen, preferring their own Ease and Sloth before the general Improvement of their Country" (p. 169)

Como hemos señalado anteriormente, la obsesión de Swift por los excrementos queda patente en toda la obra, siempre desde un punto de vista satírico:

"I went into another Chamber, but was ready to hasten back, being almost overcome with a horrible Stink. My Conductor pressed me forward, conjuring me in a Whisper to give no Offence, which would be highly resented; and therefore I durst not so much as stop my Nose. The Projector of this Cell was the most ancient Student of the Academy. His Face and Beard were of a pale Yellow; his Hands and Clothes daubed over with Filth. When I was presented to him, he gave me a close Embrace (a Compliment I could well have excused.) His Employment from his first coming into the Academy, was an Operation to reduce human Excrement to its original Food, by separating the several Parts, removing the Tincture which it receives from the Gall, making the Odour exhale, and scumming off the Saliva. He had a weekly Allowance from 
the Society, of a Vessel filled with human Ordure about the Bigness of a Bristol Barrel" (p. 171)

En cuanto a las traducciones, de nuevo es la versión de Emilio Lorenzo la más adecuada, puesto que no realiza ninguna mala interpretación del texto, siendo nuevamente la versión que resulta más fiel al texto original, como podemos comprobar a continuación:

"Pasé luego a otro aposento, pero en el acto me vi obligado a retroceder, casi vencido por un hedor insoportable. Mi guía me empujó hacia delante, pidiéndome encarecidamente al oído que evitara ofenderlos, pues lo tomarían muy a mal, asi es que no me atreví siquiera a taparme la nariz. El inventor que ocupaba esta celda era el investigador más antiguo de la Academia. Tenía el rostro y la barba amarillentos y las manos y la ropa cubiertos de mugre. Cuando me presentaron a él, me dio ún fuerte abrazo, gesto al que yo hubiera renunciado gustoso. Desde que se incorporó a la Academia su ocupación había consistido en restituir los excrementos humanos al estado primitivo de alimentos, separando sus componentes diversos, quitándoles el pigmento que reciben de la bilis, eliminando el olor y retirando las partes viscosas. Para esta empresa recibía un cupo semanal de deyecciones humanas que llenaban un recipiente del tamaño de una barrica de Bristol" (pp. 203-204)

La versión de Pedro Guardia Massó también acierta en la traducción de la primera parte, pero el traductor comete un error casi al final del párrafo cuando hace referencia al tinte, al olor y a la bilis. En la versión original, el proyectista se dedica a extraer el tinte que produce la bilis, así como el olor de dichos alimentos, mientras que en esta versión traducida, la bilis y el olor proporcionaban un tinte que era el que el proyectista tenia que extraer:

"Entré en otra habitación, pero me preparé a salir con rapidez, medio asfixiado por un hedor insoportable. Mi guia me incitó a seguir diciéndome al oído que iba a cometer una incorrección muy desagradable por lo que ni siquiera pude taparme la nariz. El inventor que vivía en esta celda era el miembro más antiguo de la academia. Su tez y barba tenían un color amarillo pálido; sus manos y vestimenta estaban cubiertos de inmundicia. Durante la presentación me endilgó un estrecho abrazo, efusión que bien podría haberse ahorrado. Desde su llegada a la academia investigaba cómo. convertir los excrementos humanos en el alimento original, aislando sus diferentes elementos, eliminando el tinte que les proporcionaba la bilis y el olor, y suprimiendo la mucosidad. Cada semana recibia de la sociedad un recipiente del tamaño de un barril de Bristol, lleno de excrementos" (p. 165) 
Por su parte, la versión de Pollux Hernúñez contiene un error de traducción bastante importante al traducir la entrada del protagonista en la habitación. Gulliver no podía ni tan siquiera taparse la nariz para evitar ofender al proyectista. Sin embargo, Hernúñez mantiene una versión totalmente contraria al texto original de Swift, al manifestar que lo único que el personaje podía hacer era taparse la nariz.

"Entré en otro cuarto, pero me heché atrás en seguida por un horrible
hedor que casi me tumba. Mi acompañante me empujó adelante
suplicándole en un susurro que no diera motivo de ofensa, que se
tomaría muy a mal, así que no me atreví más que a taparme la nariz. El
proyectista de esta celda era el investigador más antiguo de la
Academia. La cara y la barba las tenía de un amarillo pálido, las manos
y la ropa completamente embadurnadas de porquería. Cuando nos
presentaron, me dio un abrazo muy fuerte (cortesía que bien le hubiera
excusado). Su ocupación desde que llegó a la Academia era un
procedimiento para convertir el excremento humano en alimento que
originalmente es, separando los diversos componentes, retirando el
tinte que le da la bilis, haciendo que el olor se evaporara y purificándolo
de la saliva. Recibía de la Sociedad la donación semanal de un
recipiente lleno de heces humanas del tamaño de un tonel de Bristol”
(pp. 196-197)

La versión de Gárate contiene varios errores traductológicos, no tanto en el sentido de la traducción, sino en la manera de expresarse en la lengua de llegada. Algunos ejemplos de esto pueden ser el uso de las expresiones 'dejarme casi fuera de combate' o 'chorreaban inmundicias' y de la estructura 'al serle presentado a él', bastante inapropiadas en castellano:

"Penetré en otra cámara, pero me eché atrás al instante al dejarme casi fuera de combate un hedor espantoso. Mi guía me empujaba adentro, rogándome con un susurro no dar motivo de ofensa, pues se tomaría muy a mal. Así que no me atreví más que a taparme la nariz. El Proyectista de esta celda era el estudioso de mayor edad de la Academia. Su cara y su barba eran de un amarillo pálido, sus manos y sus vestidos chorreaban inmundicias. Al serle presentado a él, me dio un fuerte abrazo (cumplido del que muy a gusto habriale excusado). Su actividad desde su llegada a la academia consistia en devolver al excremento humano su prístino como alimento, separando sus diversos elementos, eliminando la pigmentación que recibe de la bilis, haciendo que desaparezca el olor, y desempumándolo de saliva. Recibía de la sociedad una asignación semanal de un recipiente repleto de porquería humana, del tamaño de una cuba de Bristol" (p. 207) 
Por otra parte, cierto es que, pese a estas modificaciones en las traducciones con respecto al sentido original del texto inglés, en todo momento se ha mantenido en todas las traducciones la sátira expresada por Swift en su viaje a Laputa.

\section{CONCLUSIÓN}

En esta tercera parte de Gulliver's Travels, la sátira textual no hace sino subrayar la sátira que Swift vierte en la Academia de Lagado. Todos los inventos ridículos que Gulliver encuenta en la Laputa tienen como fin único y primordial "improving speculative Knowledge by practical or mechanical Operations" (p. 173). Para Terry Castle, Gulliver no hace sino crear un "textbreeding machine, a device with which 'the most ignorant Person at a reasonable Charge, and with a little bodily Labour, may write Books in Philosophy, Poetry, Politicks, Law, Mathematicks and Theology, without the least Assistance from Genius or Study. He then led me to the Frame; about the Sides whereof all his Pupils stood in Ranks. It was twenty Foot Square, placed in the middle of the Room. The Superficies was composed of several bits of Wood, about the bigness of a Dye, but some larger than others. They were all linked together by slender Wires. These bits of Wood were covered on every Square with Paper pasted on them, and on these Papers were written all the Words of their Language, in their several Moods, Tenses, and Declensions, but without any Order' (p. 173)

Por su parte, las diferentes versiones de traducción han mantenido de una manera bastante fidedigna la sátira original aunque, como hemos visto, algunas de ellas no han realizado una traducción fiel al texto, sino que, en ciertas ocasiones, se ha malinterpretado el texto original, persistiendo la sátira directa, clara e incluso amarga y resentida del autor, como K. Williams manifiesta al resumir el contenido satírico de cada una de las cuatro partes:

"Gulliver's Trave/s is a direct, plain and bitter satire against the innumerable follies and corruptions in law, politics, learning, morals and religions. The first two parts are intirely political, and satirize the wars of Europe and the factions of Whig and Tory. The third part ridicules the vain pretensions of chemists, mathematicians, projectors, and the rest of that speculative tribe, who spend their time in aerial studies. The fourth part is justified, for man is viler than any Yahoo or beast"14.

${ }^{13}$ CASTLE, TERRY, "A Deconstructionist Perspective: Why the Houyhnhnms Don't Write: Swift, Satire, and the Fear of the Text", en Fox, Christopher (ed.), Gulliver's Travels: complete, authoritative text with biographical and historical contexts, critical history, and essays from five contemporary critical perspective. New York: Bedford / St. Martin's, 1995, p. 391.

${ }^{14}$ WILLIAMS, KATHLEEN, Swift: The Critical Heritage. London: Routledge, 1970, pp. 139-144. 
6. BIBLIOGRAFÍA

CASTLE, Terry, "A Deconstructionist Perspective: Why the Houyhnhnms Don't Write: Swift, Satire, and the Fear of the Text", en Fox, Christopher (ed.), Gulliver's Travels: complete, authoritative text with biographical and historical contexts, critical history, and essays from five contemporary critical perspective. New York: Bedford / St. Martin's, 1995.

DAvIS, H., Jonathan Swift. Essays on His Satire and Other Studies. New York: Oxford University, 1964.

EDDY, W.A., On Gulliver's Travels: A Critical Study. Princeton: Princeton, 1923.

EDDY, William Alfred (ed.), Swift: Satires and Personal Writings. London: OUP, (1932), 1973.

EHRENPREIS, I., Swift: The Man, His Works, and the Age. 3 vols. Cambridge: Harvard, 1962-83.

ELIAS, A. C., Swift at Moor Park: problems in biography and criticism. Philadelphia: University of Pennsylvania Press, 1982.

Fox, Christopher (ed.), Gulliver's Travels: complete, authoritative text with biographical and historical contexts, critical history, and essays from five contemporary critical perspective. New York: Bedford / St. Martin's, 1995.

MOORE, John B, "The Role of Gulliver", Modern Philology 25 (1928), pp. 46980.

MUNRO, John, "Book III of Gulliver's Travels Once More", en English Studies 49 (1968), pp. 429-36.

NiCOLSON, Marjorie and Nora Mohler, "Swift's 'Flying Island' in the Voyage to Laputa”, en Annals of Science 2 (1937), pp. 405-430.

SWIFT, Jonathan, Los viajes de Gulliver (introducción de Aránzazu Usandizaga, traducción de Begoña Gárate Ayastuy). Madrid: Alianza, 1994.

SWIFT, Jonathan, Los viajes de Gulliver (introducción, traducción y notas de Pedro Guardia Massó). Barcelona: Planeta, 1984.

SWIFT, Jonathan, Los viajes de Gulliver (traducción, introducción y notas de Emilio Lorenzo). Cáceres: Clásicos de la Lengua Inglesa en Traducción, 1998.

SWIFT, Jonathan, Los viajes de Gulliver (traducción, notas y apéndice de Pollux Hernúñez). Madrid: Anaya, 1999.

WILLIAMS, Kathleen, Swift: The Critical Heritage. London: Routledge, 1970. 